\title{
Auto-tune system using single-run relay feedback test and model-based controller design
}

\author{
Hsiao-Ping Huang *, Jyh-Cheng Jeng, Kuo-Yuan Luo \\ Department of Chemical Engineering, National Taiwan University, Taipei 10617, Taiwan, ROC
}

Received 10 February 2004; received in revised form 15 November 2004; accepted 16 November 2004

\begin{abstract}
In this paper, a systematic approach for auto-tune of PI/PID controller is proposed. A single run of the relay feedback experiment is carried out to characterize the dynamics including the type of damping behavior, the ultimate gain, and ultimate frequency. Then, according to the estimated damping behavior, the process is classified into two groups. For each group of processes, modelbased rules for controller tuning are derived in terms of ultimate gains and ultimate frequencies. To classify the processes, the estimation of an apparent deadtime is required. Two artificial neural networks (ANNs) that characterize this apparent deadtime using the ATV data are thus included to facilitate this estimation of this apparent deadtime. The model-based design for this auto-tuning makes uses of parametric models of FOPDT (i.e. first-order-plus-dead-time) and of SOPDT (i.e. second-order-plus-dead-time) dynamics. The results from simulations show that the controllers thus tuned have satisfactory results compared with those from other methods.
\end{abstract}

(C) 2004 Elsevier Ltd. All rights reserved.

Keywords: Auto-tune; Relay feedback; Artificial neural network; Apparent deadtime

\section{Introduction}

In 1984, Åström and Hägglund [1] presented a relay feedback system to generate sustained oscillation as an alternative to the conventional continuous cycling technique for controller tuning. This relay feedback test was soon referred as autotune variation (abbr. ATV) [2] via the system as shown in Fig. 1(a). Fig. 1(b) illustrates the typical response curves from this ATV system. The test provides ultimate gain $\left(k_{\mathrm{cu}}\right)$ and ultimate period $\left(P_{\mathrm{u}}\right)$ of the following to apply Ziegler-Nichols $(\mathrm{Z}-\mathrm{N})$ method for controller tuning.

\footnotetext{
* Corresponding author. Tel.: +88622363 8999; fax: +88622362 3935.

E-mail address: huanghpc@ntu.edu.tw (H.-P. Huang).
}

$$
\begin{aligned}
& k_{\mathrm{cu}}=\frac{4 h}{\pi A} \\
& \omega_{\mathrm{u}}=\frac{2 \pi}{P_{\mathrm{u}}}
\end{aligned}
$$

Controller tuning via the above mentioned ATV test is attractive, because it is operated under closed-loop and no a priori knowledge of system is needed. But, in most of auto-tune systems reported in literature, identification of model structure is generally not included. As a result, controllers are tuned using a common set of tuning formula, which are mostly $\mathrm{Z}-\mathrm{N}$ or modified $\mathrm{Z}-\mathrm{N}$ rules. However, when applying to processes that are not feasible to be controlled with such $\mathrm{Z}-\mathrm{N}$ tuned controllers, the control performance thus obtained is, in general, inferior to those tuned by model-based methods. This fact is most obvious when the processes have significantly second-order underdamped dynamics. To overcome the difficulty aforementioned, model-based controllers 

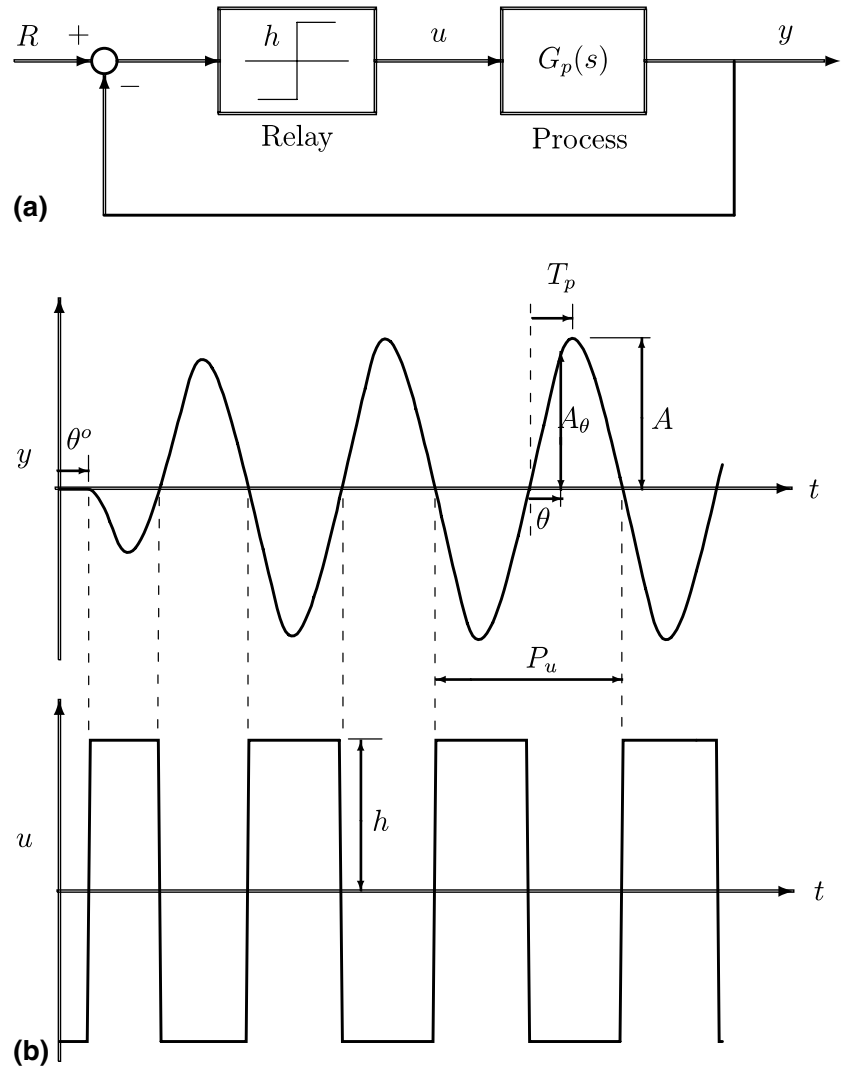

Fig. 1. (a) Block diagram of a relay feedback system. (b) Response curves in relay feedback test.

seem desirable, and, an adequate and reduced order dynamic model (usually, FOPDT or SOPDT) is necessary. On the other hand, in model-based design, PI or PID controllers will be resulted according to the dynamic models being used. For example, a FOPDT process will be controlled by either PI or PID controllers, and PID controllers will be required for SOPDT processes [3]. But, for some specific process, it may have preference on using PI controller even though the process is of SOPDT dynamics. To be more flexible for such applications, some SOPDT processes may need to have their FOPDT equivalence for controller tuning. For this reason, besides the estimation of parameter, identification of model structure in an auto-tune system is desirable. Up to the time being, quite a few works [2,4-13] trying to develop parametric models from the ATV tests have been reported. Nevertheless, according to the reported literature, the identification for parametric models needs more than one run of the ATV experiments $[4,10]$, and the model structure is usually assumed in priori. As a results, in order to explore a proper model for better control, the ATV test has to be repeated for different model structures, and, the experiments for identification will be costly.

In this paper, a systematic approach for auto-tune of $\mathrm{PI} / \mathrm{PID}$ controller is proposed. It emphasizes developing apparent dynamic model (such as: FOPDT or SOPDT) using a single run of the ATV experiment. With a few constant cycles from this ATV experiment, parameters including the steady-state gain $\left(k_{\mathrm{p}}\right)$, an apparent deadtime $(\theta)$, ultimate gain, and ultimate frequency are estimated. The estimated apparent deadtime together with some generalized ATV responses of standard SOPDT processes is used to characterize the damping behavior the process. In each groups, different model-based tuning formulas in terms of ultimate gain and ultimate frequency are provided. These formulas are derived, using parameterization of FOPDT or SOPDT dynamics, to shape the loop transfer function of the system to a given and desired form. In the following, it is assumed that zero offset is a common specification of control. For this reason, tuning rules including both PI and PID types of controllers are considered.

The remainder of this paper is organized as follows. The controllers design including tuning strategy is given in Section 2. Process identification and classification for controller tuning using ATV test are presented in Section 3 . The auto-tune procedures and several examples used to illustrate the effectiveness of this proposed method are shown in Section 4 followed by the conclusion.

\section{Controllers design}

The step response of an open-loop stable process can be roughly classified into two categories. One has monotonic response and the other has underdamped oscillations. The transfer functions that can be used to represent these two types of dynamics can be different. For example, the former can usually be represented using an FOPDT model, while the latter has to be represented by a model of underdamped SOPDT. These two types of model are:

\section{- FOPDT model}

$G_{\mathrm{p}}(s)=\frac{k_{\mathrm{p}} \mathrm{e}^{-\theta s}}{\tau s+1}$

- Underdamped SOPDT model

$G_{\mathrm{p}}(s)=\frac{k_{\mathrm{p}} \mathrm{e}^{-\theta s}}{\tau^{2} s^{2}+2 \tau \zeta s+1}$

Nevertheless, it is possible that two different types of transfer function mentioned have the same $k_{\mathrm{cu}}$ and $\omega_{\mathrm{u}}$. If $\mathrm{Z}-\mathrm{N}$ types of controller settings are to be applied, it is obvious that if it works with acceptable performance to the FOPDT type processes, it may be unacceptable to the SOPDT ones. In other words, to be applied to different types of dynamic processes, tuning rules should be changed according to the type of dynamics. As one can see the dynamic responses of systems tuned by $\mathrm{Z}-\mathrm{N}$ or $\mathrm{T}-\mathrm{L}$ [14] methods from Fig. 2, the system with a smaller 

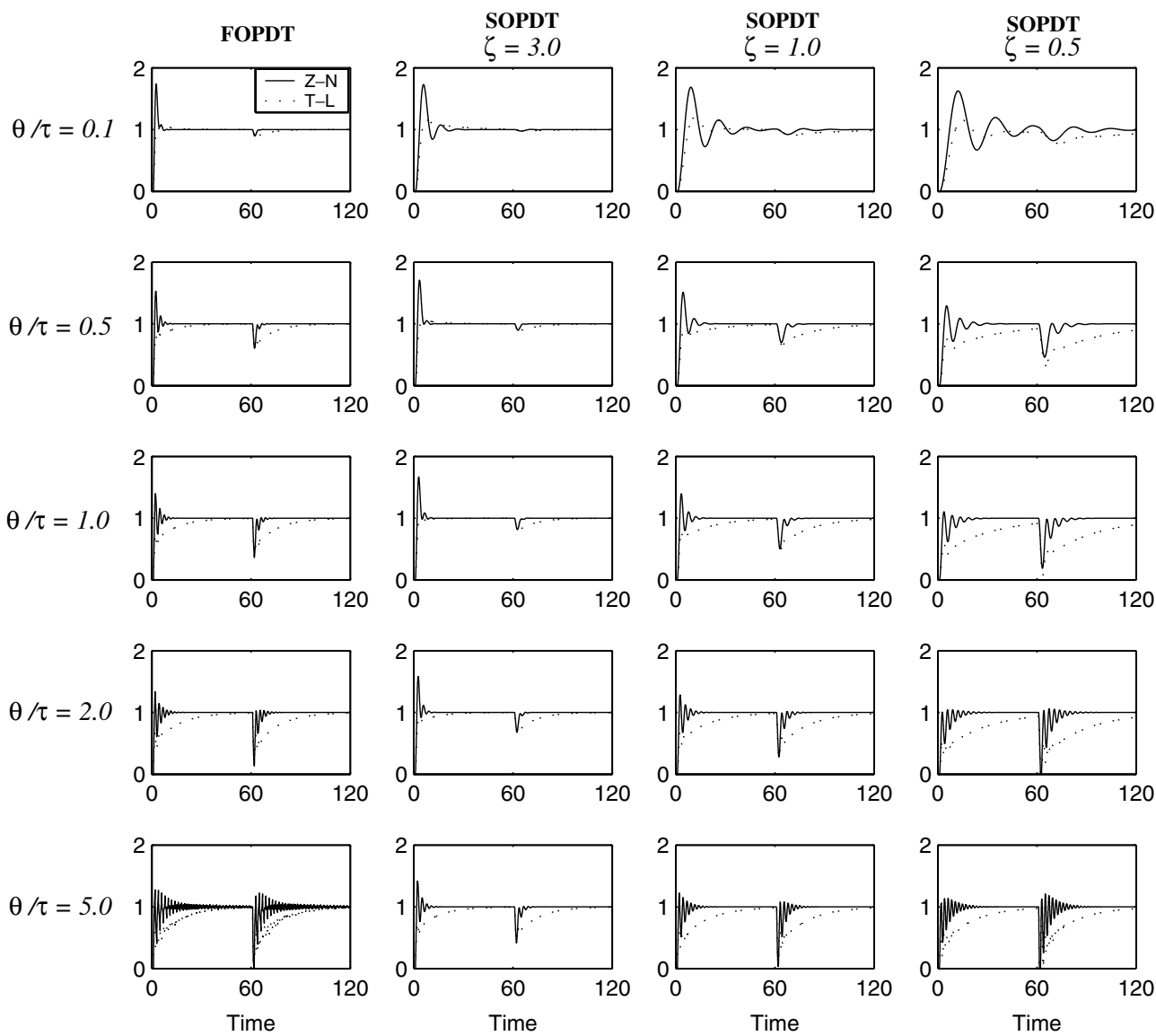

Fig. 2. Comparisons of control responses for different dynamics using $\mathrm{Z}-\mathrm{N}$ and $\mathrm{T}-\mathrm{L}$ tuning rules.

damping factor has much difference in performance from that of FOPDT process, compared with that of overdamped system to the FOPDT process. This reflects a simple common fact that overdamped and some of underdamped processes can be described approximately by an FOPDT model. For this reason, the damping factor of the dynamic system is adopted as a measure to differentiate the type of dynamics for controller tuning. If this is to be the case, it is found that in order to have an FOPDT model to represent an SOPDT process, it is necessary that the ultimate loop gain, $K_{\mathrm{u}}$ (i.e. $k_{\mathrm{p}} k_{\mathrm{cu}}$ ), should be greater than one. This result is obvious due to the following facts. The FOPDT parameters in terms of ultimate gain and ultimate frequency can be written as:

$$
\begin{aligned}
\tau & =\frac{\sqrt{K_{\mathrm{u}}^{2}-1}}{\omega_{\mathrm{u}}} \\
\theta & =\frac{\pi-\tan ^{-1}\left(\sqrt{K_{\mathrm{u}}^{2}-1}\right)}{\omega_{\mathrm{u}}}
\end{aligned}
$$

Thus, if $K_{\mathrm{u}} \leqslant 1$, there will be trivial or non-real $\tau$ and $\theta$ from Eq. (4). According to the definition of $K_{\mathrm{u}}$ in terms of an SOPDT process in frequency domain, the following is concluded: $\left(1-\omega_{\mathrm{u}}^{2} \tau^{2}\right)^{2}+\left(2 \omega_{\mathrm{u}} \tau \zeta\right)^{2}=K_{\mathrm{u}}^{2}$

In Eq. (5), there must have at least one non-negative and real solution for $\omega_{\mathrm{u}}$, so it is concluded (see the proof in the Appendix A) that: $\omega_{\mathrm{u}}>0$ and $K_{\mathrm{u}}>1$, if $\zeta>$ $1 / \sqrt{2} \approx 0.71$.

According to the fact presented above, it is obvious that model-based controllers design based on an FOPDT model or an underdamped SOPDT model is desirable to implement the auto-tune of the PI/PID controllers. In the following, designs of these controllers will be given.

\subsection{Tuning PI/PID controllers based on FOPDT model}

The synthesis of PI/PID controller using an FOPDT parameterization is considered as follows. The controller is used to compensate the process to provide a loop transfer function (abbr. LTF) of the following standard form:

$G_{\text {loop }}(s)=\frac{k_{\mathrm{o}}}{\theta} \frac{(1+a \theta s)}{s\left(1+\tau_{\mathrm{f}} s\right)} \mathrm{e}^{-\theta s}$

The use of loop transfer function in the form of Eq. (6) has been explained in a late work of Huang and Jeng [15]. A system having such an LTF with $k_{\mathrm{o}}$ and $a$ 
properly selected has satisfactory control performance as well as reasonable stability robustness.

According to the standard form, the synthesis of PID controller is rather straightforward. The resulting PID controller is given as following:

$G_{\mathrm{c}}(s)=k_{\mathrm{c}}^{\prime}\left(1+\frac{1}{\tau_{\mathrm{R}}^{\prime} s}\right)\left(\frac{1+\tau_{\mathrm{D}}^{\prime} s}{1+\tau_{\mathrm{f}} s}\right)$

where

$$
\begin{aligned}
k_{\mathrm{c}}^{\prime} & =\frac{k_{\mathrm{o}} \tau}{k_{\mathrm{p}} \theta} \\
\tau_{\mathrm{R}}^{\prime} & =\tau \\
\tau_{\mathrm{D}}^{\prime} & =a \theta
\end{aligned}
$$

As mentioned, $k_{\mathrm{o}}$ and $a$ are two adjustable parameters, and the filter time constant, $\tau_{\mathrm{f}}$, is to be taken arbitrarily small (e.g. $0.05 \tau_{\mathrm{D}}^{\prime}$ ). From Fig. 3, it is found that these two parameters, $k_{\mathrm{o}}$ and $a$, are closely related to the ro-
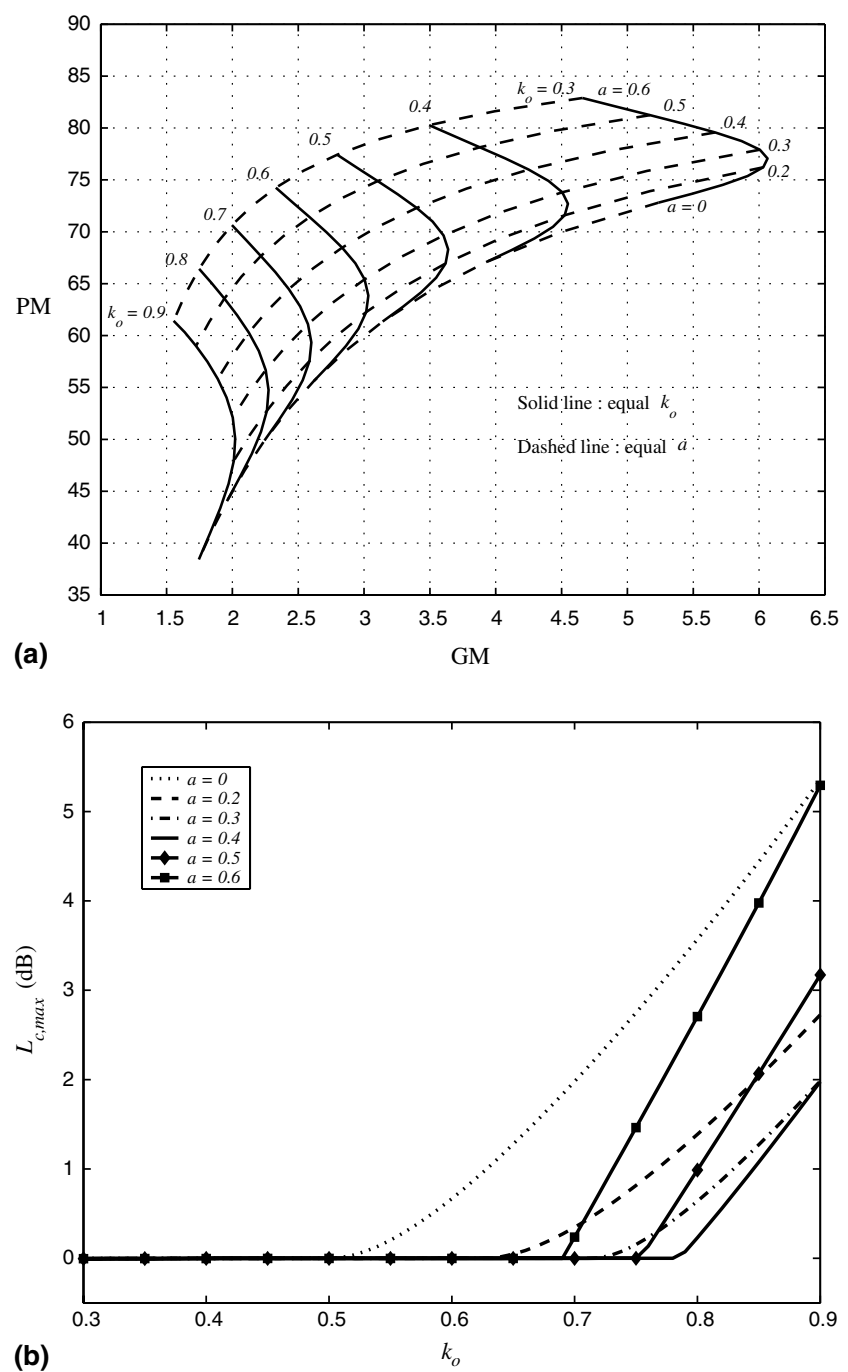

Fig. 3. (a) Gain margin (GM), phase margin (PM), (b) $L_{\mathrm{c}, \max }$ of proposed control systems. bust stability and performance of the system. The defaulted values of $k_{\mathrm{o}}$ and $a$ can be given as 0.65 and 0.4 , respectively, to provide a gain margin of 2.7 , a phase margin of $65^{\circ}$ and a $L_{\mathrm{c}, \max }$ of $0 \mathrm{~dB}$.

On the other hand, the synthesis of PI controller is not so intuitive since, for FOPDT model, the LTF of Eq. (6) cannot be exactly achieved by using a PI controller. Therefore, the PI controller is derived to make the resulting LTF as an approximation of its standard form, and is given as following:

$G_{\mathrm{c}}(s)=k_{\mathrm{c}}\left(1+\frac{1}{\tau_{\mathrm{R}} S}\right)$

where

$$
\begin{aligned}
k_{\mathrm{c}} & =\frac{k_{\mathrm{o}} \tau_{\mathrm{R}}}{k_{\mathrm{p}} \theta} \\
\tau_{\mathrm{R}} & =0.9 \tau+a \theta
\end{aligned}
$$

Here, the defaulted values of $k_{\mathrm{o}}$ and $a$ are taken as 0.55 and 0.4 , respectively.

Since the ultimate gain and ultimate frequency can be obtained directly from ATV test. By substituting Eq. (4) into Eq. (8), the PID parameters that are originally given in terms of the FOPDT parameterization can be re-written as:

$$
\begin{aligned}
k_{\mathrm{c}}^{\prime} & =\frac{k_{\mathrm{o}} \sqrt{K_{\mathrm{u}}^{2}-1}}{k_{\mathrm{p}}\left[\pi-\tan ^{-1}\left(\sqrt{K_{\mathrm{u}}^{2}-1}\right)\right]} \\
\tau_{\mathrm{R}}^{\prime} & =\frac{\sqrt{K_{\mathrm{u}}^{2}-1}}{\omega_{\mathrm{u}}} \\
\tau_{\mathrm{D}}^{\prime} & =a\left[\frac{\pi-\tan ^{-1}\left(\sqrt{K_{\mathrm{u}}^{2}-1}\right)}{\omega_{\mathrm{u}}}\right]
\end{aligned}
$$

Similarly, substituting Eq. (4) into Eq. (10), the PI tuning formula can be written as:

$$
\begin{aligned}
& k_{\mathrm{c}}=\frac{k_{\mathrm{o}}}{k_{\mathrm{p}}}\left[\frac{0.9 \sqrt{K_{\mathrm{u}}^{2}-1}}{\left[\pi-\tan ^{-1}\left(\sqrt{K_{\mathrm{u}}^{2}-1}\right)\right]}+a\right] \\
& \tau_{\mathrm{R}}=\frac{1}{\omega_{\mathrm{u}}}\left[0.9 \sqrt{K_{\mathrm{u}}^{2}-1}+a\left[\pi-\tan ^{-1}\left(\sqrt{K_{\mathrm{u}}^{2}-1}\right)\right]\right]
\end{aligned}
$$

Notice that to use these above equations for controller tuning, a value greater than one for $K_{\mathrm{u}}$ is necessary.

\subsection{Tuning PID controllers based on underdamped SOPDT model}

As mentioned, a significantly underdamped process (i.e., $\zeta \leqslant 1 / \sqrt{2}$ ) is not feasible to be represented by the 
FOPDT model. Generally speaking, it is not appropriate to control this underdamped dynamics using a PI controller, unless it is carefully tuned. This is because use of PI controller has strong influence on the stability of the system. To retain the stability robustness as well as its performance, the following loop transfer function is preferred:

$G_{\text {loop }}(s)=\frac{k_{\mathrm{o}}}{\theta} \frac{\mathrm{e}^{-\theta s}}{s\left(1+\tau_{\mathrm{f}} s\right)}$

In other words, a PID controller of the following is recommended:

$G_{\mathrm{c}}(s)=k_{\mathrm{c}}\left(1+\frac{1}{\tau_{\mathrm{R}} S}+\tau_{\mathrm{D}} S\right)\left(\frac{1}{1+\tau_{\mathrm{f}} S}\right)$

where

$k_{\mathrm{c}}=\frac{k_{\mathrm{o}}(2 \tau \zeta)}{k_{\mathrm{p}} \theta}$

$\tau_{\mathrm{R}}=2 \tau \zeta$

$\tau_{\mathrm{D}}=\frac{\tau}{2 \zeta}$

In this case, $k_{\mathrm{o}}$ is the only free parameter that can be tuned to define the performance and robustness of the system. The reason for choosing the loop transfer function mentioned is that loop transfer function of Eq. (13) is commonly resulted from the IMC design. In Fig. 3, the curves corresponding to $a=0$ show the gain margin, phase margin, and $L_{\mathrm{c}, \max }$ of such a system using $k_{\mathrm{o}}$ as parameter. The defaulted value of $k_{\mathrm{o}}$ is taken as 0.5 .

In terms of the ultimate gain and ultimate frequency, the values of $\tau$ and $\zeta$ can be computed from the following equations (see Appendix B for detailed derivation):

$\tau=\frac{\sqrt{1+K_{\mathrm{u}} \cos \left(\omega_{\mathrm{u}} \theta\right)}}{\omega_{\mathrm{u}}}$

$\zeta=\frac{K_{\mathrm{u}} \sin \left(\omega_{\mathrm{u}} \theta\right)}{2 \sqrt{1+K_{\mathrm{u}} \cos \left(\omega_{\mathrm{u}} \theta\right)}}$

As a result, the PID parameters of Eq. (15) are given as:

$k_{\mathrm{c}}=\frac{k_{\mathrm{o}} K_{\mathrm{u}} \sin \left(\omega_{\mathrm{u}} \theta\right)}{k_{\mathrm{p}} \omega_{\mathrm{u}} \theta}$

$\tau_{\mathrm{R}}=\frac{K_{\mathrm{u}} \sin \left(\omega_{\mathrm{u}} \theta\right)}{\omega_{\mathrm{u}}}$

$\tau_{\mathrm{D}}=\frac{1+K_{\mathrm{u}} \cos \left(\omega_{\mathrm{u}} \theta\right)}{\omega_{\mathrm{u}} K_{\mathrm{u}} \sin \left(\omega_{\mathrm{u}} \theta\right)}$

Thus, if we have ultimate gain, $k_{\mathrm{cu}}$, and ultimate frequency, $\omega_{\mathrm{u}}$, of the given process, the parameters of the
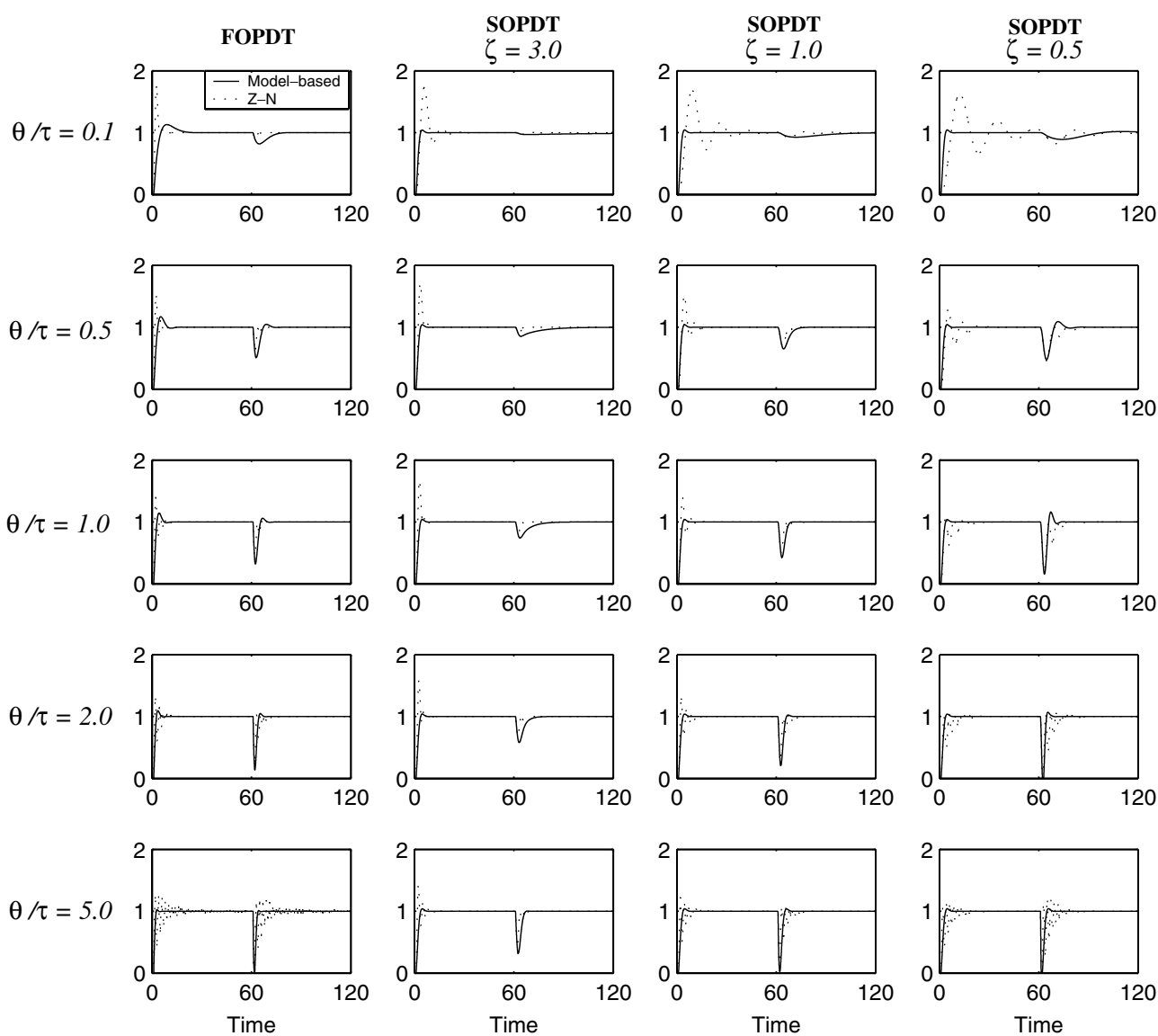

Fig. 4. Comparisons of control responses using model-based and $\mathrm{Z}-\mathrm{N}$ tuning rules. 
PI or PID controllers can be computed based on either Eqs. (12), (11), or Eq. (17). However, as one can seen in Eq. (17), the tuning formula need to know the values of $k_{\mathrm{p}}$ and the apparent deadtime $\theta$ in advance. The estimation for $k_{\mathrm{p}}$ and $\theta$ will be given in the next section.

Notice that the proposed model-based formula in terms of an FOPDT parameterization is different from the IMC-PID controller of Chien and Fruehauf [3]. There is no use of approximation for $\mathrm{e}^{-\theta s}$ in deriving the controller and, when deadtime of the system is normalized to be one, the resulting system responds almost in the same way regardless of the value of $\theta / \tau$.

\subsection{Performance and tuning strategy}

Fig. 4 shows the comparisons of the responses of controlled FOPDT and SOPDT processes, with deadtime equaling one, using the presented model-based or the $\mathrm{Z}-\mathrm{N}$ tuning rules. Notice that the model-based controller gives uniformly the same shape of set-point responses for all SOPDT processes regardless the ratio of $\theta / \tau$, and has an overshoot less than $10 \%$. The fact that the modelbased controller has superior control performance to the $\mathrm{Z}-\mathrm{N}$ controller can be justified based on the results in the same figure. The setpoint tracking performance of model-based controller is much superior, while the disturbance performance is still compatible with $\mathrm{Z}-\mathrm{N}$ con- troller, which is recognized suitable for disturbance rejection. Moreover, the proposed model-based control system is more robust than $\mathrm{Z}-\mathrm{N}$ tuned system. As has been observed, the system with $\mathrm{Z}-\mathrm{N}$ controller has either excessive overshoot or oscillations, which is not desirable in the control practice. The reasons why $\mathrm{Z}-\mathrm{N}$ yields excessive responses can partially be explained by looking into the tuning parameters of both methods for FOPDT processes, as shown in Fig. 5. The Z-N method provides too aggressive $k_{\mathrm{c}}$ almost for all values of $\theta / \tau$. The $\tau_{R}$ and $\tau_{D}$ settings of $Z-N$ are also too aggressive, compared with those of model-based ones, when $\theta / \tau$ is small. But, on the other hand, they are slightly conservative when $\theta / \tau$ is large. To prevent such over responses, in literature, Tyreus and Luyben [14] suggested that the proportional gain, reset time, and derivative time of PID controller are multiplied by a factor of $0.76,4.4$, and 1.27 , respectively. But, even modifying in this way, the presented model-based control is still superior to the T-L control, as shown in their comparisons in Fig. 6.

On the other hand, as has been mentioned, processes with SOPDT dynamics, which are overdamped or slightly underdamped, are sometimes identified with FOPDT models for controller design, and the criterion will be $\zeta>(1 / \sqrt{2})$. Thus, if uses of Eq. (11) and of Eq. (17) are discriminated using $\zeta=1 / \sqrt{2}$ as a bound-
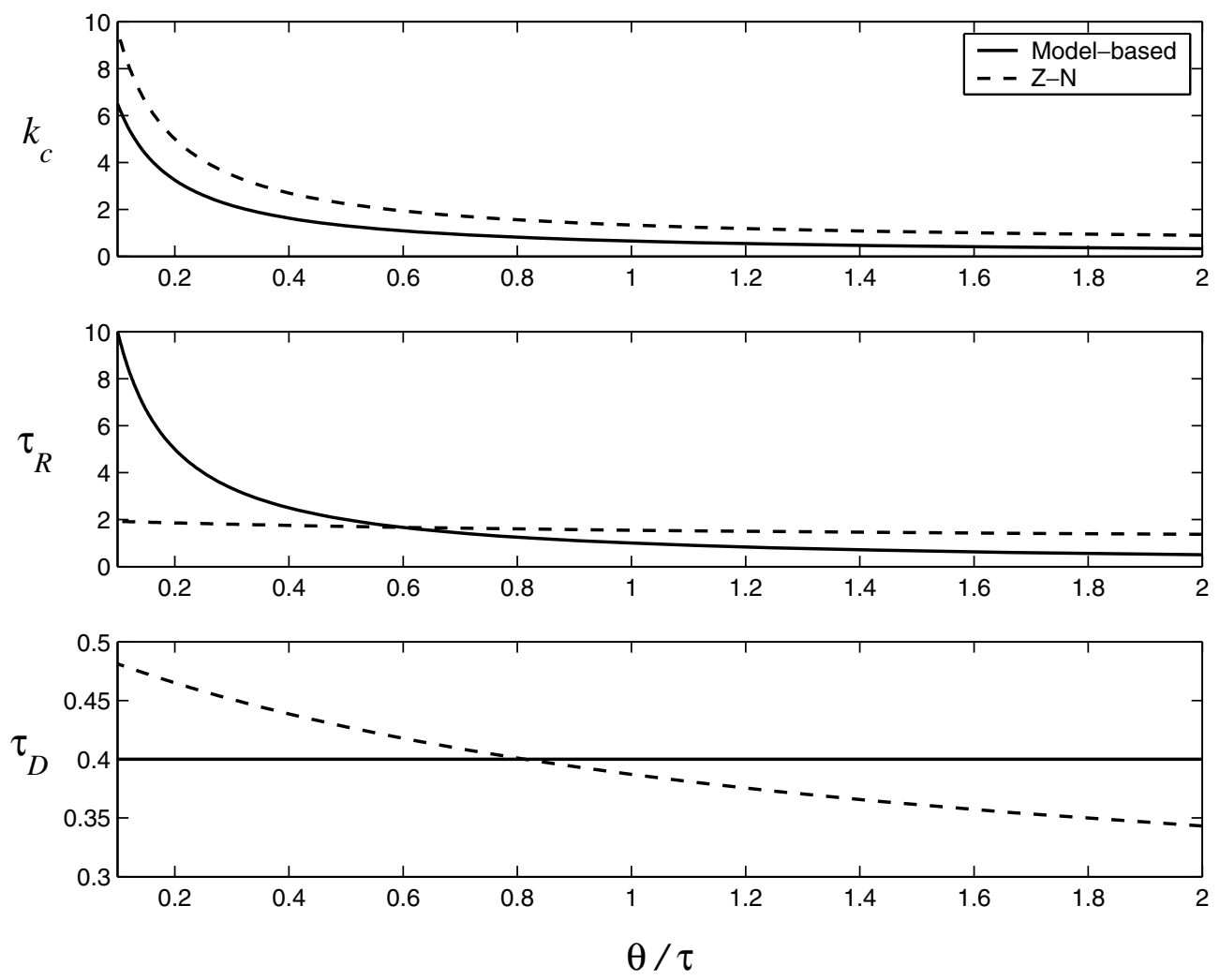

Fig. 5. Comparisons of model-based and Z-N controller settings for FOPDT processes $(\theta=1)$. 

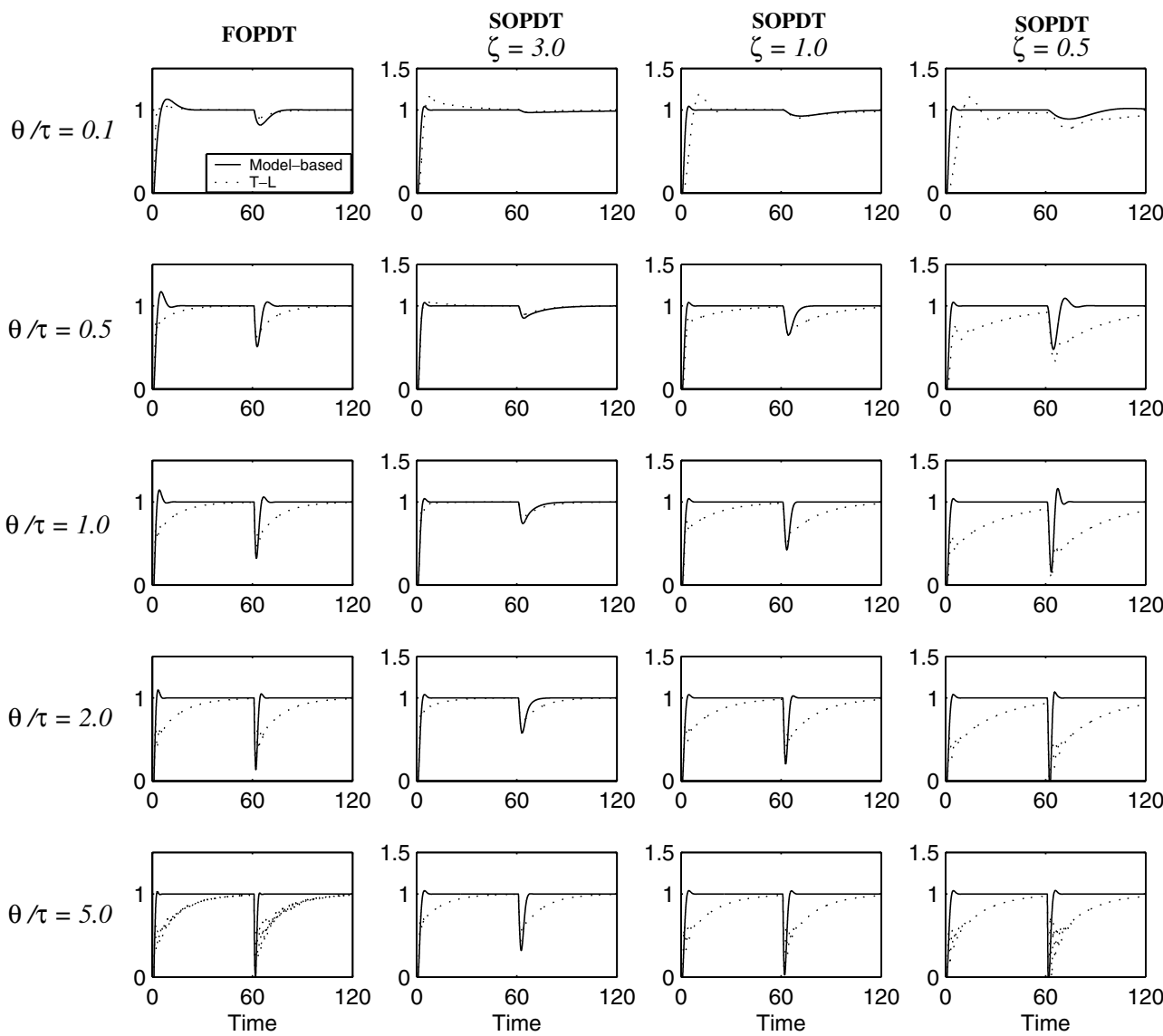

Fig. 6. Comparisons of control responses using model-based and $\mathrm{T}-\mathrm{L}$ tuning rules.

ary, Fig. 7 shows the comparison of the performances. It can be seen that other than the cases of small $\theta / \tau$, an SOPDT process that has a damping factor greater than $1 / \sqrt{2}$ have no significant difference in performances when either equations are applied. In case that an SOPDT process has $\zeta>1 / \sqrt{2}$, then it belongs to one group of which Eqs. (11) and (12) can be applied, and either PI or PID controller can be used. On the other hand, if $\zeta \leqslant 1 / \sqrt{2}$, the process belongs to the other group of which Eq. (17) is applied for computing controller parameters so that only PID controller is used. The proposed model-based tuning strategy and design method can be summarized as shown in Fig. 8 .

\section{Process identification using ATV test}

To apply the model-based controllers derived in the previous section, reduced order dynamic models such as FOPDT of Eq. (2), and, as SOPDT of Eq. (3) are required. The advantages of using ATV for identification is obvious. The most important reason is that the test can be carried out under a closed-loop. The resulting cycling period and magnitude are adopted as estimation value of ultimate period and ultimate gain due to the theory of describing function. With these two experimental data, it is not sufficient for identifying parametric models more complicated than the FOPDT one. As a result, modifications to the relay $[7,8,12]$, or, repeated experiment with altered system blocks $[4,10]$ to obtained more data to solve identification for parametric models have been reported in literature. But, since experiments for identification are usually expensive and subjected to unknown disturbances, it is most desirable to complete the identification within a few constant cycles in one single run. In the following, the method of identification proposed is guided by this requirement.

As shown in Fig. 1(a), an ATV system consists of process, $G_{\mathrm{p}}$, and a relay controller in a single closedloop. The relay controller provides output at two levels (i.e. $+h$ or $-h$ ) as on-off actions. The controlled process output starts with transient oscillations after a pure deadtime, $\theta^{\mathrm{o}}$, and then develops constant cycling with magnitude $A$ and period $P_{\mathrm{u}}$. Notice that $A$ and $P_{\mathrm{u}}$ are used in the auto-tune system of $\AA$ Aström and Hägglund [1]. Furthermore, one height $A_{\theta}$ and the time $T_{\mathrm{p}}$ associated with the constant cycles are also indicated in Fig. 1(b). All these quantities measured from the ATV test are governed by the dynamic model and the properties of the relay. In other words, they can be expressed as: 

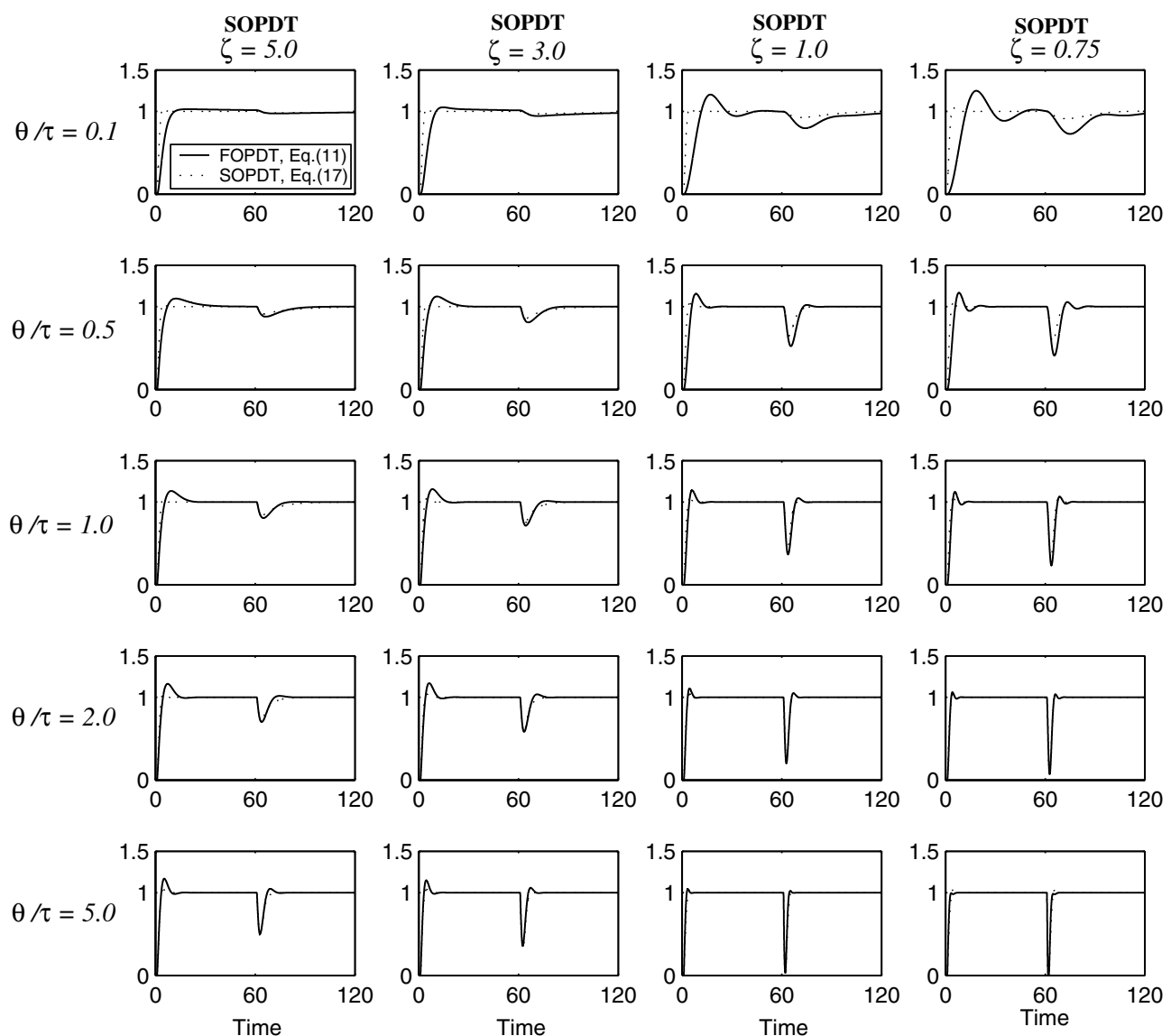

Fig. 7. Comparisons of control responses using tuning rules of Eqs. (11) and (17) for SOPDT process with $\zeta>1 / \sqrt{2}$.

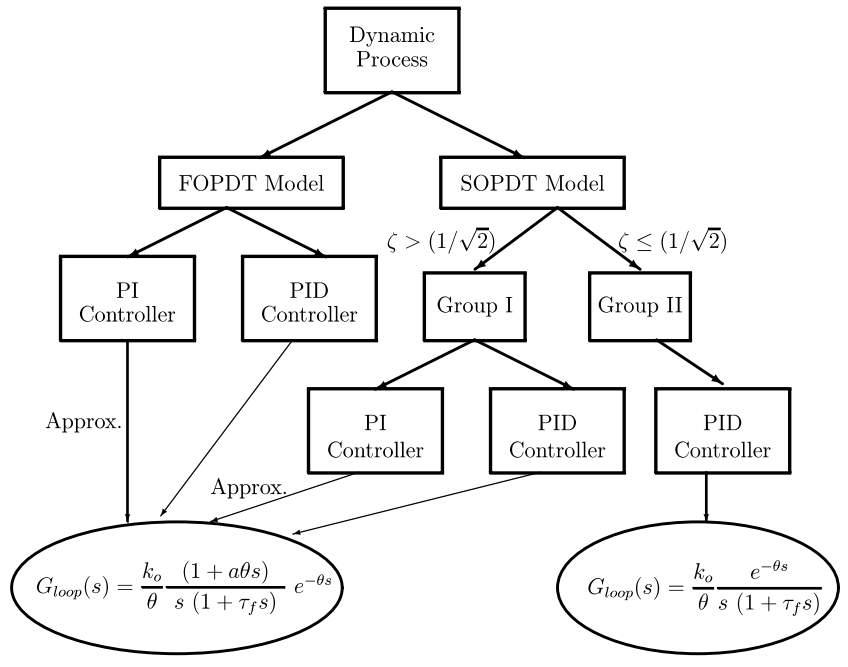

Fig. 8. Tuning strategy for the model-based auto-tune system.

$$
\begin{aligned}
& \frac{A}{k_{\mathrm{p}} h}=f_{1}(\bar{\tau}) \quad \text { or } \quad f_{1}(\bar{\tau}, \zeta) \\
& \frac{P_{\mathrm{u}}}{\theta}=f_{2}(\bar{\tau}) \quad \text { or } \quad f_{2}(\bar{\tau}, \zeta)
\end{aligned}
$$

$$
\begin{aligned}
& \frac{A_{\theta}}{A}=f_{3}(\bar{\tau}) \quad \text { or } \quad f_{3}(\bar{\tau}, \zeta) \\
& \frac{\theta}{T_{\mathrm{p}}}=f_{4}(\bar{\tau}) \quad \text { or } \quad f_{4}(\bar{\tau}, \zeta)
\end{aligned}
$$

where $\bar{\tau}$ represents $\tau / \theta$. The equations given above describe the dynamics of the ATV response in time domain.

Two more equations which correspond to Eqs. (18) and (19) derived from frequency domain are:

$\omega_{\mathrm{u}} \theta+\tan ^{-1}\left(\frac{2 \omega_{\mathrm{u}} \tau \zeta}{1-\omega_{\mathrm{u}}^{2} \tau^{2}}\right)=\pi$

and

$\frac{k_{\mathrm{p}}}{\sqrt{\left(1-\omega_{\mathrm{u}}^{2} \tau^{2}\right)^{2}+4 \omega_{\mathrm{u}}^{2} \tau^{2} \zeta^{2}}}=\frac{1}{k_{\mathrm{cu}}}$

where $\omega_{\mathrm{u}}$ is taken as $2 \pi / P_{\mathrm{u}}$. Theoretically, provided that $k_{\mathrm{p}}$ and $k_{\mathrm{cu}}$ are given, the identification problem to find an reduced order SOPDT model can be solved by finding $\tau, \zeta$, and $\theta$ that fit Eqs. (18)-(21) or Eqs. (20)-(23) in the sense of least-squares.

Notice that the relay feedback system is non-linear, and solutions from the system to have the relations of 
Eqs. (18)-(21) need extensive efforts. Furthermore, inverse of Eqs. (18), (19) and of Eqs. (20), (21) is needed for identification. Because explicit functional forms for Eqs. (18)-(21) could be too complicated to be of practical value for the purposes aforementioned, the functional relations are easier to be modeled using empirical forms for general use. In the following, a systematic approach enhanced by using the ANNs (artificial neural networks) for identification will be presented.

\subsection{Estimation of $k_{p}$ and $k_{c u}$ from ATV experiment}

To estimate $k_{\mathrm{p}}$, the experimental ATV test is started with a temporal disturbance to either the set-point or the process input (i.e. $u$ ) for a short period of time and restored back to its origin. The disturbance introduced has two main purposes. One is to initialize the relay feedback control, the other one is to generate data for computing the steady-state process gain, $k_{\mathrm{p}}$. The estimation is made along the ATV test in one run as the following.

Let $y^{\mathrm{I}}$ and $u^{\mathrm{I}}$ designate the integrations of $y$ and $u$ from the very beginning of the experiment in one run. That is:

$$
\begin{aligned}
& y^{\mathrm{I}}(t)=\int_{0}^{t} y(\tau) \mathrm{d} \tau \\
& u^{\mathrm{I}}(t)=\int_{0}^{t} u(\tau) \mathrm{d} \tau
\end{aligned}
$$

For some $t>T$ when $y$ in an ATV test starts to oscillate with constant period and amplitude, $y^{\mathrm{I}}$ and $u^{\mathrm{I}}$ will have similar cycling responses. Typical curves of $y^{\mathrm{I}}$ and $u^{\mathrm{I}}$ are shown in Fig. 9. Let $y_{\mathrm{av}}^{\mathrm{I}}$ and $u_{\mathrm{av}}^{\mathrm{I}}$ designate the average heights of constant cycles of $y^{\mathrm{I}}$ and $u^{\mathrm{I}}$, respectively. The value of $k_{\mathrm{p}}$ can be estimated as:

$k_{\mathrm{p}}=\frac{y_{\mathrm{av}}^{\mathrm{I}}}{u_{\mathrm{av}}^{\mathrm{I}}}$
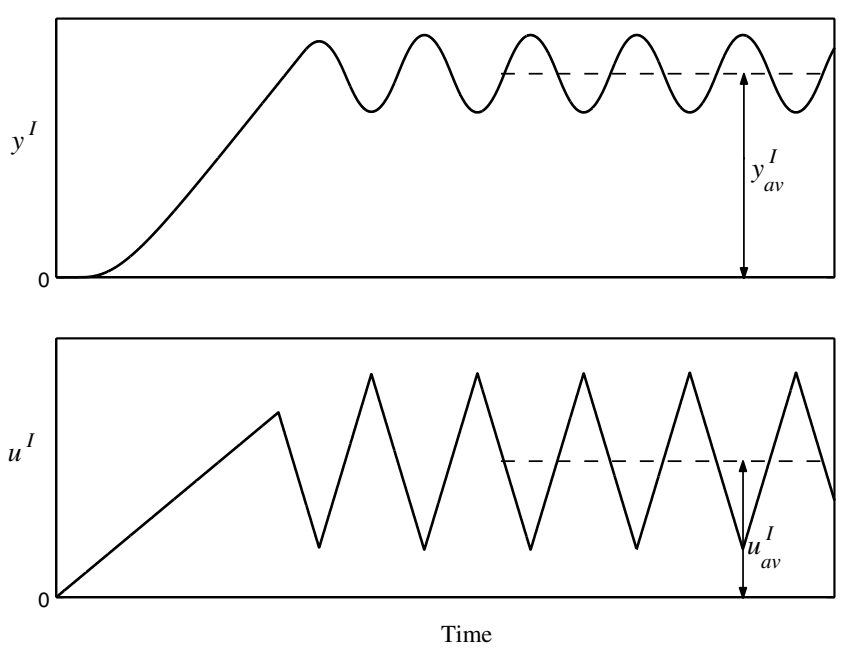

Fig. 9. Typical curves of $y^{\mathrm{I}}$ and $u^{\mathrm{I}}$ in proposed ATV test.
Due to the use of describing function for estimation, the ultimate gain computed from Eq. (1) is subjected to error, which may, sometimes, be as high as $20 \%$ [4]. For tuning purpose, estimation of the ultimate gain $k_{\mathrm{cu}}$ with improved accuracy is desirable. Shen et al. [8] had used saturation-relay feedback method to give better accuracy for $k_{\mathrm{cu}}$. Here, in order to give more accurate $k_{\mathrm{cu}}$ by original ATV test, the following estimation step is considered. Since $u(t)$ and $y(t)$ are periodic with period $P_{\mathrm{u}}$, the frequency response of $G_{\mathrm{p}}$ at ultimate frequency $\omega_{\mathbf{u}}$ are calculated via the following equation $[9,16]$ :

$G_{\mathrm{p}}\left(\mathrm{j} \omega_{\mathrm{u}}\right)=\frac{\int_{t_{0}+P_{\mathrm{u}}}^{t_{0}} y(t) \mathrm{e}^{-\mathrm{j} \omega_{\mathrm{u}} t} \mathrm{~d} t}{\int_{t_{0}}^{t_{0}+P_{\mathrm{u}}} u(t) \mathrm{e}^{-\mathrm{j} \omega_{\mathrm{u}} t} \mathrm{~d} t}$

where $t_{0}$ is taken as any time instant in a constant cycle. Then, the ultimate gain can be computed as:

$k_{\mathrm{cu}}=\frac{1}{\left|G_{\mathrm{p}}\left(\mathrm{j} \omega_{\mathrm{u}}\right)\right|}$

\subsection{Estimation of apparent deadtime}

The apparent deadtime is the deadtime appearing in an FOPDT or SOPDT model that approximates best the higher order process. As a result, in general, this apparent deadtime differs from its true deadtime (designated as $\theta^{\circ}$ ) that used to be detected at the very beginning period of the test. Features in the cycling response can be used to distinguish the FOPDT from SOPDT dynamics. For example, in case of FOPDT process, $T_{\mathrm{p}}$ equals $\theta^{\circ}$ or $\theta$. The same equality does not apply to the SOPDT case. In an ATV test, two measured quantities, $A_{\theta} / A$ and $\theta / T_{\mathrm{p}}$, are used to characterize the effect of the apparent deadtime. These two quantities, as mentioned earlier, are functions of $\bar{\tau}$ and $\zeta$. To explore their functional relations, simulations of ATV tests on the standard SOPDT processes covering wide range of $\bar{\tau}$ (in dimensionless form) and $\zeta$ are carried out. Results of $A_{\theta} / A$ and $\theta / T_{\mathrm{p}}$ for underdamped SOPDT processes are plotted as a graph as shown in Fig. 10(a), using $\bar{\tau}$ and $\zeta$ as parameters. Each pair of the two measured quantities corresponding to a point in the graph, where a specific pair of values for $\bar{\tau}$ and $\zeta$ can be found. As shown in the figure, it is difficult to read $\bar{\tau}$ and $\zeta$ from the figure. In order to make each curve in Fig. 10(a) more readable, the coordinate is rotated through the following transformation.

$$
\begin{aligned}
& X=\frac{\theta}{T_{\mathrm{p}}} \cos (\pi / 3)-\frac{A_{\theta}}{A} \sin (\pi / 3) \\
& Y=\frac{\theta}{T_{\mathrm{p}}} \sin (\pi / 3)+\frac{A_{\theta}}{A} \cos (\pi / 3)
\end{aligned}
$$

The results are plotted in Fig. 10(b).

Furthermore, for applying these data more efficiently, two artificial neural networks are constructed to 

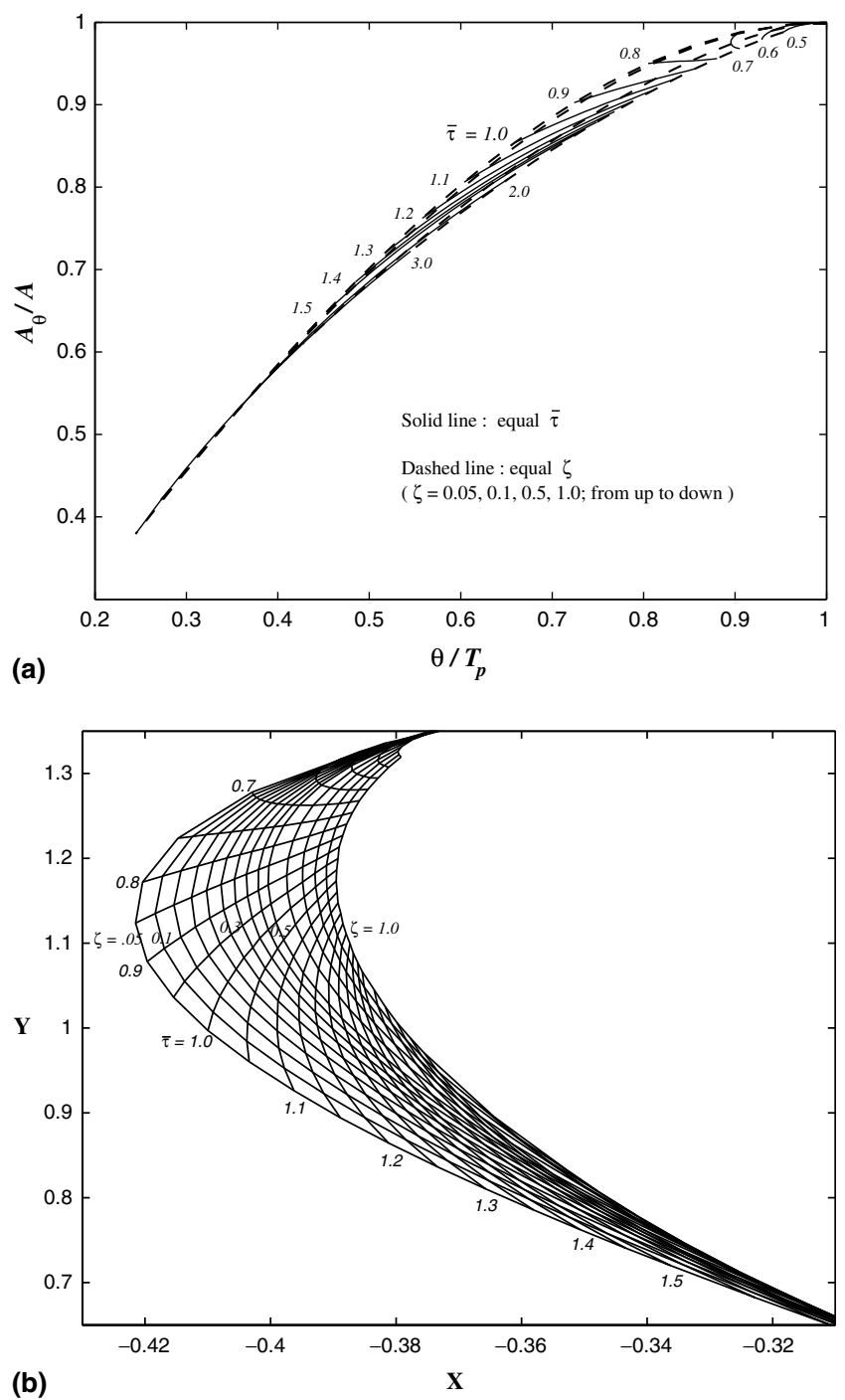

Fig. 10. Results of $A_{\theta} / A$ and $\theta / T_{\mathrm{p}}$ in the ATV test for SOPDT processes. (a) Original and (b) transformed.

represent the database. The network architecture is as shown in Fig. 11. These two neural networks are fed with $X$ and $Y$, and compute $\bar{\tau}$ and $\zeta$, respectively. Each net-

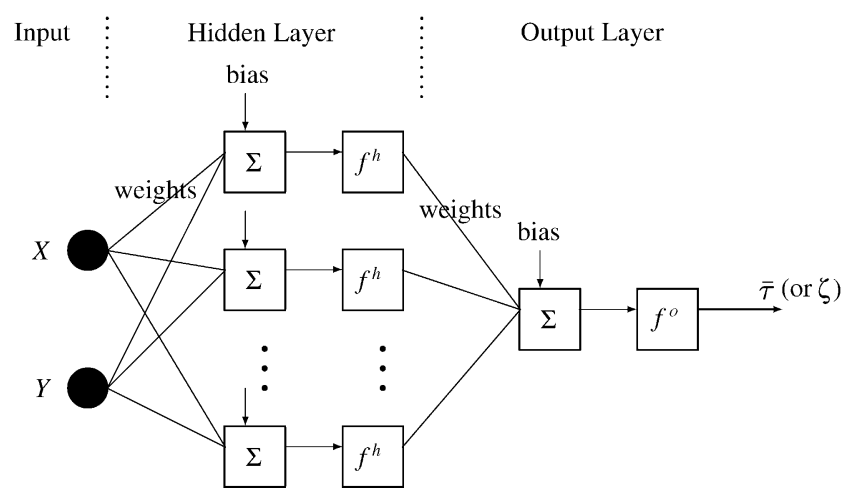

Fig. 11. Neural network architecture. work consists of feedforward net with one input layer, one hidden layer, and one output layer. The sigmoid functions, $f^{\mathrm{h}}$ and $f^{\mathrm{o}}$, are used in an error backpropagation technique to minimize the error between prediction and target values. They are hyperbolic tangent activation function and linear activation function in the hidden neurons and output neuron, respectively. It can be written as:

$\bar{\tau}($ or $\zeta)=\sum_{i=1}^{N_{\mathrm{h}}} w_{i}^{\mathrm{o}} f^{\mathrm{h}}\left[m_{i}\right]+b^{\mathrm{o}}$

$m_{i}=w_{i, X}^{\mathrm{h}} X+w_{i, Y}^{\mathrm{h}} Y+b_{i}^{\mathrm{h}}$

where $N_{\mathrm{h}}$ is the number of neurons in the hidden layer, $w_{i}^{\mathrm{o}}$ and $b^{\mathrm{o}}$ are the weights and the bias of hidden-to-output layer, and $w_{i, X}^{\mathrm{h}}, w_{i, Y}^{\mathrm{h}}$ and $b_{i}^{\mathrm{h}}$ are the weights and the bias of input-to-hidden layer. Thus, $m_{i}$ is the summation of all products, between inputs and input-to-hidden weights in the input layer, and bias. After emulation, the number of neurons in the hidden layer for both neural networks is found as 6 (i.e. $N_{\mathrm{h}}=6$ ), and the values of each weights and bias are given in Tables 1 and 2 .

With these two networks, estimation of the apparent deadtime can be proceeded. The estimation makes uses of the phase criterion in Eq. (22) and the ANNs aforementioned. Notice that three unknowns are required to satisfy at least four functional relations (i.e. Eqs. (18)-(21) or Eqs. (20)-(23)), and iterative check is thus necessary to find the solution in a least-squares sense. By solving Eqs. (20)-(22), a unique solution for $\tau$, $\zeta$, and $\theta$ can be found. But, the resulting solution may not necessarily satisfy Eq. (23). The final solution needs further iterative procedures. To satisfy the extra Eq. (23), it is found that manifold in the space of $\tau$ and $\zeta$ results from the relation of the following:

$\tan ^{-1}\left(\frac{2 \omega_{\mathrm{u}} \tau \zeta}{1-\omega_{\mathrm{u}}^{2} \tau^{2}}\right)=\pi-\omega_{\mathrm{u}} \theta$

Table 1

The values of weights and bias in the neural network for $\hat{\bar{\tau}}$

\begin{tabular}{lrrrrrl}
\hline$i$ (neuron) & 1 & \multicolumn{1}{l}{3} & \multicolumn{1}{l}{4} & \multicolumn{1}{l}{5} & 6 \\
\hline$w_{i, X}^{\mathrm{h}}$ & 0.852 & -0.153 & 0.262 & 1.034 & 1.138 & 2.047 \\
$w_{i, Y}^{\mathrm{h}}$ & 2.224 & -1.739 & -1.497 & 0.770 & 0.742 & 3.297 \\
$b_{i}^{\mathrm{h}}$ & 6.315 & 19.036 & -12.627 & -0.448 & 16.765 & 7.029 \\
$w_{i}^{\mathrm{o}}$ & -3.288 & 2.138 & 1.507 & -0.247 & 3.381 & 5.338 \\
$b^{\mathrm{o}}$ & & \multicolumn{7}{c}{-10.295} \\
\hline
\end{tabular}

Table 2

The values of weights and bias in the neural network for $\hat{\zeta}$

\begin{tabular}{llrrrrl}
\hline$i$ (neuron) & 1 & 2 & 3 & 4 & 5 & 6 \\
\hline$w_{i, X}^{\mathrm{h}}$ & -1.081 & -5.963 & 0.341 & 0.119 & 1.054 & 0.838 \\
$w_{i, Y}^{\mathrm{h}}$ & -2.031 & -4.630 & 1.228 & -0.995 & 0.485 & 0.957 \\
$b_{i}^{\mathrm{h}}$ & 10.115 & 8.540 & -9.777 & -13.576 & 7.398 & 7.751 \\
$w_{i}^{\mathrm{h}}$ & -3.056 & 14.445 & -1.703 & 1.028 & 3.455 & 1.903 \\
$b^{\mathrm{o}}$ & \multicolumn{7}{c}{-10.960} \\
\hline
\end{tabular}


With $\omega_{\mathrm{u}}$ and $\theta$ being fixed, there are many pairs of $\tau$ and $\zeta$ that satisfy Eq. (22), and one of these pairs would make Eq. (23) satisfied. However, in this proposed auto-tune system, only the apparent deadtime of the process is required, estimation of the exact values of $\tau$ and $\zeta$ can be skipped except a complete process model is desired. To avoid confusing, the values of $\bar{\tau}$ and $\zeta$ used in the estimation of $\theta$ are designated as $\hat{\bar{\tau}}$ and $\hat{\zeta}$, respectively, in the following text. They can be viewed as two auxiliary variables for estimating $\theta$, but not necessarily the actual parameters of SOPDT model.

With the theory presented above, the algorithm for the estimation of apparent deadtime can be made in a simpler way as the following:

(1) Starting from a guessed value of $\theta$, which is initially taken as $\theta^{\circ}$.

(2) The values of $X$ and $Y$ are calculated and fed into two networks. Then, parameters $\hat{\bar{\tau}}$ and $\hat{\zeta}$ are computed, and check if Eq. (22) holds. The procedure proceeds iteratively by increasing the guess value of $\theta$ until $\theta$ equals $T_{\mathrm{p}}$.

(3) When, at certain value of guessed $\theta$, Eq. (22) holds true, the resulting guess value $\theta$ is taken as the estimated value of apparent deadtime.

(4) If, until the guessed value of $\theta$ exceeds $T_{\mathrm{p}}$ and no candidate solution is found, the underdamped SOPDT model is not good for describing the dynamics of the process. As a result, model of FOPDT or overdamped SOPDT should be used and the controller tuning can be based on FOPDT parameterization directly.

Notice that the proposed method mentioned is quite suitable for processes which can be represented by FOPDT or SOPDT model. Other than the two types of models mentioned (e.g. processes with zeros), this method still works for the cases where the zeros are small and can be taken as extra lead time or as extra pure deadtime.

\subsection{Classification for controller tuning}

As mentioned previously, as long as the process can be modeled with an SOPDT model whose damping factor is greater than a value of $(1 / \sqrt{2})$, this process can be tuned with Eq. (11) or Eq. (12). In addition, from the ATV test, it provides $A$ and $P_{\mathrm{u}}$ which are functions of $\tau$ and $\zeta$. As shown in Fig. 12(a), there are curve lines of constant $\zeta$ 's. The one that corresponds to a damping factor of $1 / \sqrt{2}$ is found to be represented by the following equation (curve $\mathrm{A}$ ):

$\Omega=0.465 \Lambda^{3}-2.002 \Lambda^{2}+0.958 \Lambda-0.007$

where $\Omega=\log \left(A / k_{\mathrm{p}} h\right)$ and $\Lambda=\log \left(P_{\mathrm{u}} / \theta\right)$. Similarly, the curve for FOPDT processes are found to be (curve B):
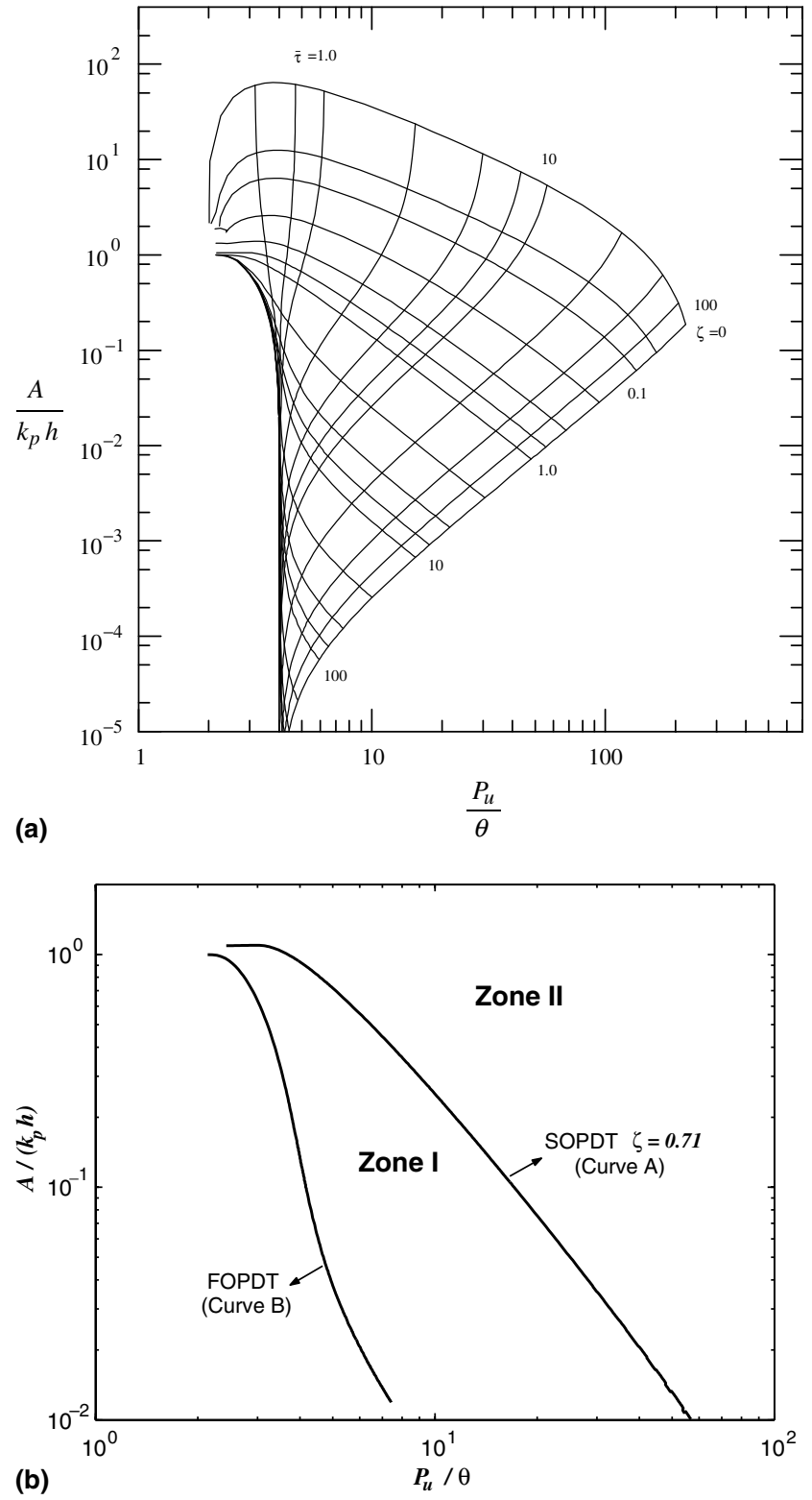

Fig. 12. Results of $A /\left(k_{\mathrm{p}} h\right)$ and $P_{\mathrm{u}} / \theta$ in the ATV test for FOPDT and SOPDT processes. (a) Generalized and (b) simplified.

$\Omega=30.931 \Lambda^{3}-56.784 \Lambda^{2}+29.032 \Lambda-4.505$

These curve lines divide the graph of Fig. 12(a) into two zones as shown in 12(b). One is between the curves A and $\mathrm{B}$ (i.e. Zone I) that represents the feasible region for using parameterization of FOPDT, and the other region above curve A (i.e. Zone II) that represents the feasible region to use parameterization of underdamped SOPDT. Thus, once $k_{\mathrm{p}}$ and the apparent deadtime $\theta$ are obtained, the normalized value of $A /\left(k_{\mathrm{p}} h\right)$ and $P_{\mathrm{u}} /$ $\theta$ can be calculated. Then, compare the resulting value of $\log \left(A / k_{\mathrm{p}} h\right)$ with the one calculated from Eq. (31). If the former is smaller, then this process is classified into the group (Group I) where Eq. (11) or Eq. (12) can be applied to tune PID or PI controllers. Otherwise, the 
process is classified into the other group (Group II) where Eq. (17) will be used for tuning PID controllers. In general, the $\log \left(A / \mathrm{k}_{\mathrm{p}} h\right)$ and $\log \left(P_{\mathrm{u}} / \theta\right)$ pair will lie above the curve B. In case of it lies beneath that curve, models other than the FOPDT or SOPDT should be considered.

\section{Auto-tune procedures with illustrated examples}

To perform the auto-tune, the relay feedback of the ATV test is initialized by a short period of manual disturbance. The process input and output responses are recorded until they have constant cycling, and the following procedure steps are taken:

(1) Compute $k_{\mathrm{p}}$ from Eq. (25) and $k_{\mathrm{cu}}$ from Eq. (27).

(2) Take $\theta^{\circ}$ as an initial guess for $\theta$ and compute values of $\hat{\bar{\tau}}$ and $\hat{\zeta}$ using two networks. The resulting parameters are used to check if Eq. (22) is satisfied.

(3) The above procedure step is proceeded by increasing gradually the guessed value of $\theta$ along the response cycle until Eq. (22) is satisfied. When Eq. (22) is satisfied, the final guessed $\theta$ is the apparent deadtime.

(4) Having the value of apparent deadtime, the process is classified by comparing the value of $A$ / $\left(k_{\mathrm{p}} h\right)$ from ATV test with the one calculated by Eq. (31).

(5) If the process belongs to Group I, Eq. (11) or Eq. (12) is used for tuning PID or PI controllers, respectively.

(6) If the process belongs to Group II, Eq. (17) is used to tune a PID controller.

Notice that, in case of significantly overdamped dynamics, the trajectory of the $\left(\theta / T_{\mathrm{p}}, A_{\theta} / A\right)$ will be crowded at the lower edge of the graph in Fig. 10(a).
In that case, the process can be classified into Group I directly without estimating the apparent deadtime.

In order to illustrate the above auto-tune procedures, a few examples are given as following. Notice that, in each example, the amplitude of relay output is normalized as one (i.e. $h=1$ ). First, the following process is considered:

$G_{\mathrm{p}}(s)=\frac{\mathrm{e}^{-1.5 s}}{(s+1)^{5}}$

As the first example, the auto-tune procedures will be described in detail. To initialize the ATV test, the system is set on manual and give a small step change of magnitude 0.2 to the process input for a short period of 10 , and then switch back to perform ATV test. After sustained oscillation is attained, the information obtained from ATV test are summarized in Table 3. The steadystate gain is computed from Eq. (25) as $k_{\mathrm{p}}=$ $y_{\mathrm{av}}^{\mathrm{I}} / u_{\mathrm{av}}^{\mathrm{I}}=0.565 / 0.560=1.01$. The ultimate gains $k_{\mathrm{cu}}$ computed from Eq. (1) and Eq. (27) are 1.773 and 1.809 , respectively. Notice that the actual $k_{\mathrm{cu}}$ for this process is 1.796. The possible apparent deadtime $\theta$ ranges from $\theta^{\circ}=1.5$ to 3.194 (i.e. the value of $T_{\mathrm{p}}$ ), and several points of transformed $\left(\theta / T_{\mathrm{p}}, A_{\theta} / A\right)$ in this range are plotted as shown in Fig. 13(a). The corresponding $\hat{\bar{\tau}}$ and $\hat{\zeta}$ for each guess of $\theta$ used to check if Eq. (22) is satisfied can be found from neural networks. The associated error $\epsilon$ defined as

$\epsilon=\pi-\omega_{\mathrm{u}} \theta-\tan ^{-1}\left(\frac{2 \omega_{\mathrm{u}} \hat{\tau} \hat{\zeta}}{1-\omega_{\mathrm{u}}^{2} \hat{\tau}^{2}}\right)$

to represent the degree-of-satisfaction of Eq. (22) is plotted as shown in Fig. 13(b). Among these points, Eq. (22) is found to be most satisfied by the one with $\theta=2.91$. Therefore, the apparent deadtime in terms of SOPDT model for this process is taken as 2.91 . For process classification, locate $\left(P_{\mathrm{u}} / \theta, A / k_{\mathrm{p}} h\right)=(4.172,0.718)$ on Fig. 14,

Table 3

Results of ATV test and estimated parameters

\begin{tabular}{|c|c|c|c|c|c|c|c|c|c|c|}
\hline \multirow[t]{2}{*}{ Example } & \multirow[t]{2}{*}{ Process } & \multicolumn{4}{|c|}{ ATV test } & \multicolumn{5}{|c|}{ Estimated parameters } \\
\hline & & $A$ & $P_{\mathrm{u}}$ & $\theta^{\mathrm{o}}$ & $T_{\mathrm{p}}$ & $k_{\mathrm{p}}$ & $k_{\mathrm{cu}}^{\mathrm{a}}$ & $k_{\mathrm{cu}}^{\mathrm{b}}$ & $k_{\mathrm{cu}}{ }^{\mathrm{c}}$ & $\theta(\mathrm{SOPDT})$ \\
\hline 1 & $\frac{\mathrm{e}^{-1.5 s}}{(s+1)^{5}}$ & 0.718 & 12.140 & 1.5 & 3.194 & 1.01 & 1.773 & 1.809 & 1.796 & 2.91 \\
\hline 2 & $\frac{\mathrm{e}^{-3 s}}{\left(s^{2}+10 s+1\right)(s+1)^{2}}$ & 0.315 & 16.998 & 3.0 & 4.316 & 1.01 & 4.039 & 4.314 & 4.273 & 3.62 \\
\hline 3 & $\frac{\mathrm{e}^{-2.2 s}}{\left(4 s^{2}+2.8 s+1\right)(s+1)^{2}}$ & 0.876 & 14.392 & 2.2 & 3.770 & 1.02 & 1.454 & 1.480 & 1.472 & 3.44 \\
\hline 4 & $\frac{(0.5 s+1) \mathrm{e}^{-s}}{(s+1)^{2}(2 s+1)}$ & 0.353 & 6.562 & 1.0 & 4.316 & 1.01 & 3.607 & 3.741 & 3.776 & 1.32 \\
\hline 5 & $\frac{(-0.5 s+1) \mathrm{e}^{-s}}{(s+1)^{2}(2 s+1)}$ & 0.575 & 9.200 & 1.0 & 2.290 & 1.00 & 2.214 & 2.336 & 2.340 & 2.07 \\
\hline 6 & $\frac{\mathrm{e}^{-2 s}}{\left(9 s^{2}+2.4 s+1\right)(s+1)}$ & 1.171 & 15.900 & 2.0 & 3.926 & 1.00 & 1.087 & 1.109 & 1.110 & 2.72 \\
\hline
\end{tabular}

\footnotetext{
${ }^{\text {a }}$ Computed from Eq. (1).

b Computed from Eq. (27).

c Actual value.
} 

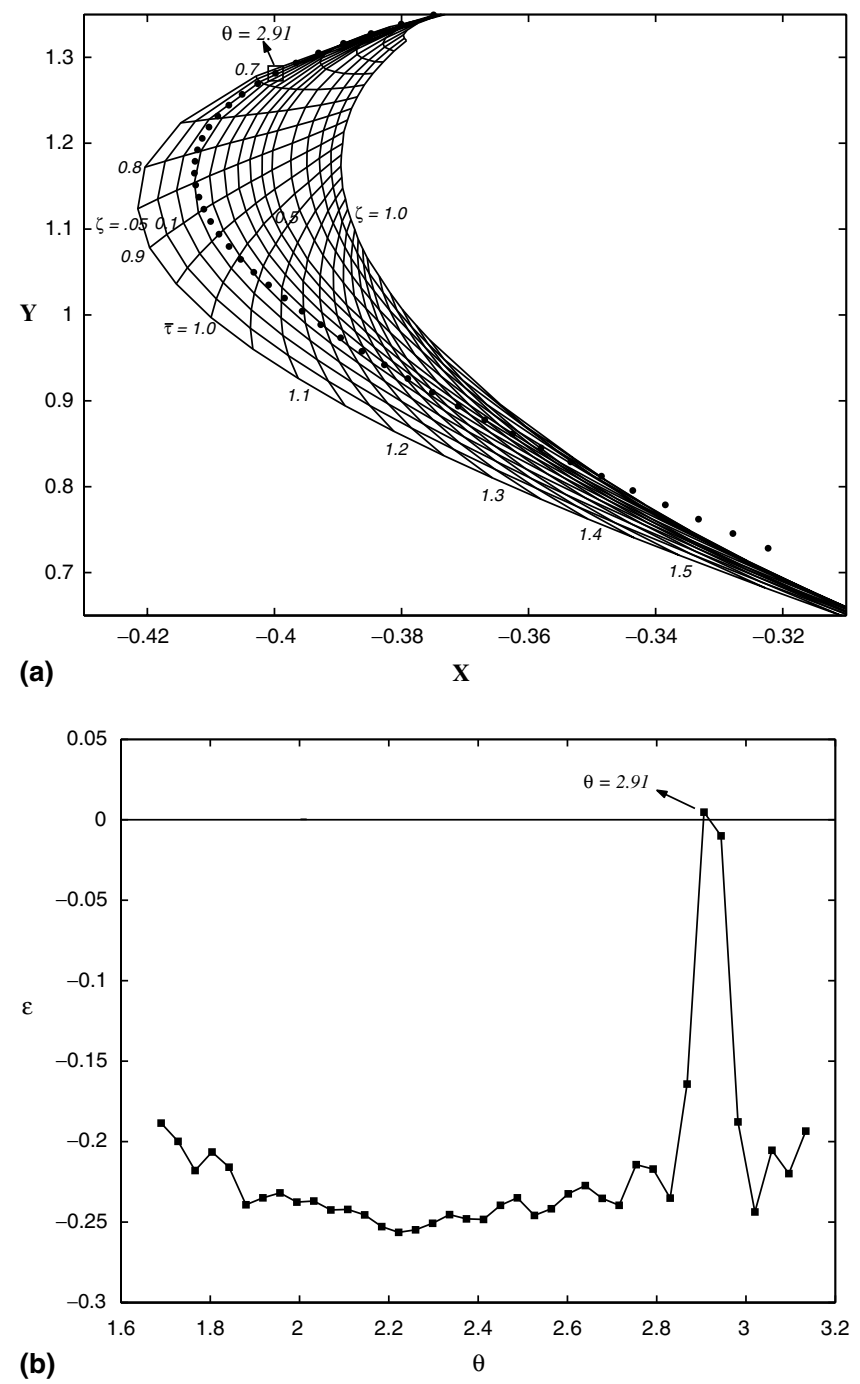

Fig. 13. Estimation of apparent deadtime for example 1 (a) trajectory of transformed $\left(\theta / T_{\mathrm{p}}, A_{\theta} / A\right)$ and (b) associated error of each point.

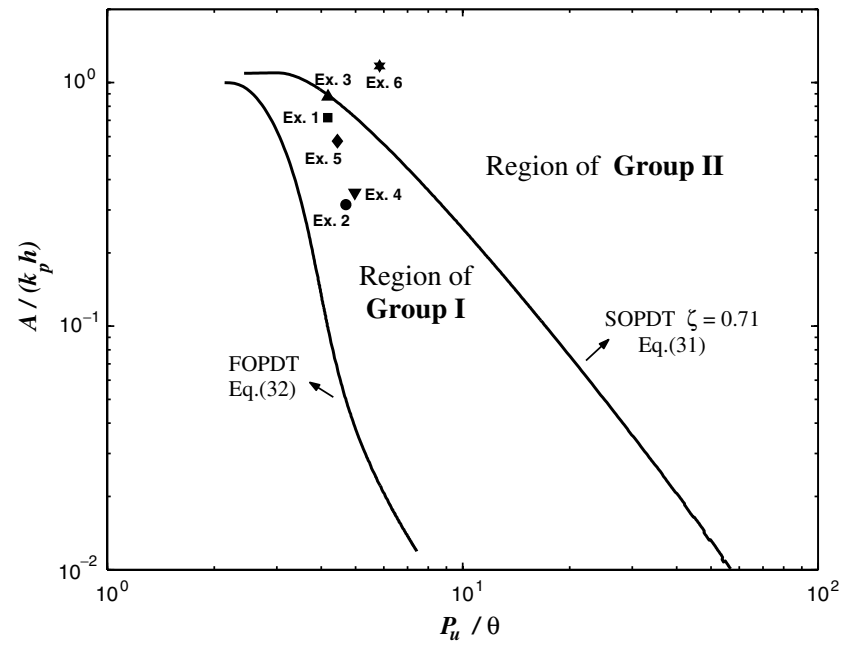

Fig. 14. Results of process classification.

and it can be seen that this point falls into the region of Group I. Therefore, Eq. (12) or Eq. (11) is used for tuning PI or PID controller. The resulting PI/PID settings are compared with $\mathrm{Z}-\mathrm{N}$ settings as listed in Table 4. Furthermore, the control results as shown in Fig. 15(a) are found more satisfactory than those of $\mathrm{Z}-\mathrm{N}$ tuning.

Simulations are also carried out for other five examples, including processes with zero. The results of ATV test and some estimated parameters are presented in Table 3. The process classification and resulting PI/PID tuning are listed in Table 4. For comparison, the PI/ PID parameters of $\mathrm{Z}-\mathrm{N}$ method are also given in Table 4. The control results using proposed model-based method and Z-N method are shown in Fig. 15(b)-(f). In Fig. 15(f), in addition to the $\mathrm{Z}-\mathrm{N}$ response, the proposed method is compared with two others from a conventional auto-tune system using IMC rules. In applying

Table 4

Classification and controller tuning of illustrated examples

\begin{tabular}{|c|c|c|c|c|c|c|c|}
\hline \multirow[t]{2}{*}{ Example } & \multirow[t]{2}{*}{ Classification } & \multirow[t]{2}{*}{ Tuning method } & \multicolumn{3}{|l|}{ PID } & \multicolumn{2}{|l|}{ PI } \\
\hline & & & $k_{\mathrm{c}}^{\prime}$ & $\tau_{\mathrm{R}}^{\prime}$ & $\tau_{\mathrm{D}}^{\prime}$ & $k_{\mathrm{c}}$ & $\tau_{\mathrm{R}}$ \\
\hline \multirow[t]{2}{*}{1} & Group I & Proposed & 0.458 & 2.955 & 1.662 & 0.566 & 5.321 \\
\hline & & $\mathrm{Z}-\mathrm{N}$ & 1.043 & 6.070 & 1.518 & 0.806 & 10.115 \\
\hline \multirow[t]{2}{*}{2} & Group I & Proposed & 1.514 & 11.473 & 1.950 & 1.371 & 12.276 \\
\hline & & $\mathrm{Z}-\mathrm{N}$ & 2.376 & 8.499 & 2.125 & 1.836 & 14.165 \\
\hline \multirow[t]{2}{*}{3} & Group I & Proposed & 0.314 & 2.590 & 2.103 & 0.455 & 4.434 \\
\hline & & $\mathrm{Z}-\mathrm{N}$ & 0.855 & 7.196 & 1.799 & 0.661 & 11.993 \\
\hline \multirow[t]{2}{*}{4} & Group I & Proposed & 1.275 & 3.805 & 0.768 & 1.189 & 4.193 \\
\hline & & $\mathrm{Z}-\mathrm{N}$ & 2.122 & 3.281 & 0.820 & 1.640 & 5.468 \\
\hline \multirow[t]{3}{*}{5} & Group I & Proposed & 0.681 & 3.090 & 1.179 & 0.739 & 3.961 \\
\hline & & $\mathrm{Z}-\mathrm{N}$ & 1.302 & 4.600 & 1.150 & 1.006 & 7.667 \\
\hline & & & $k_{\mathrm{c}}$ & $\tau_{\mathrm{R}}$ & $\tau_{\mathrm{D}}$ & & \\
\hline \multirow[t]{4}{*}{6} & Group II & Proposed & 0.454 & 2.468 & 3.964 & & \\
\hline & & $\mathrm{Z}-\mathrm{N}$ & 0.512 & 9.938 & 1.590 & & \\
\hline & & $\operatorname{IMC}(\lambda=\theta)$ & 0.499 & 1.994 & 5.717 & & \\
\hline & & $\operatorname{IMC}(\lambda=\tau)$ & 0.371 & 1.994 & 5.717 & & \\
\hline
\end{tabular}



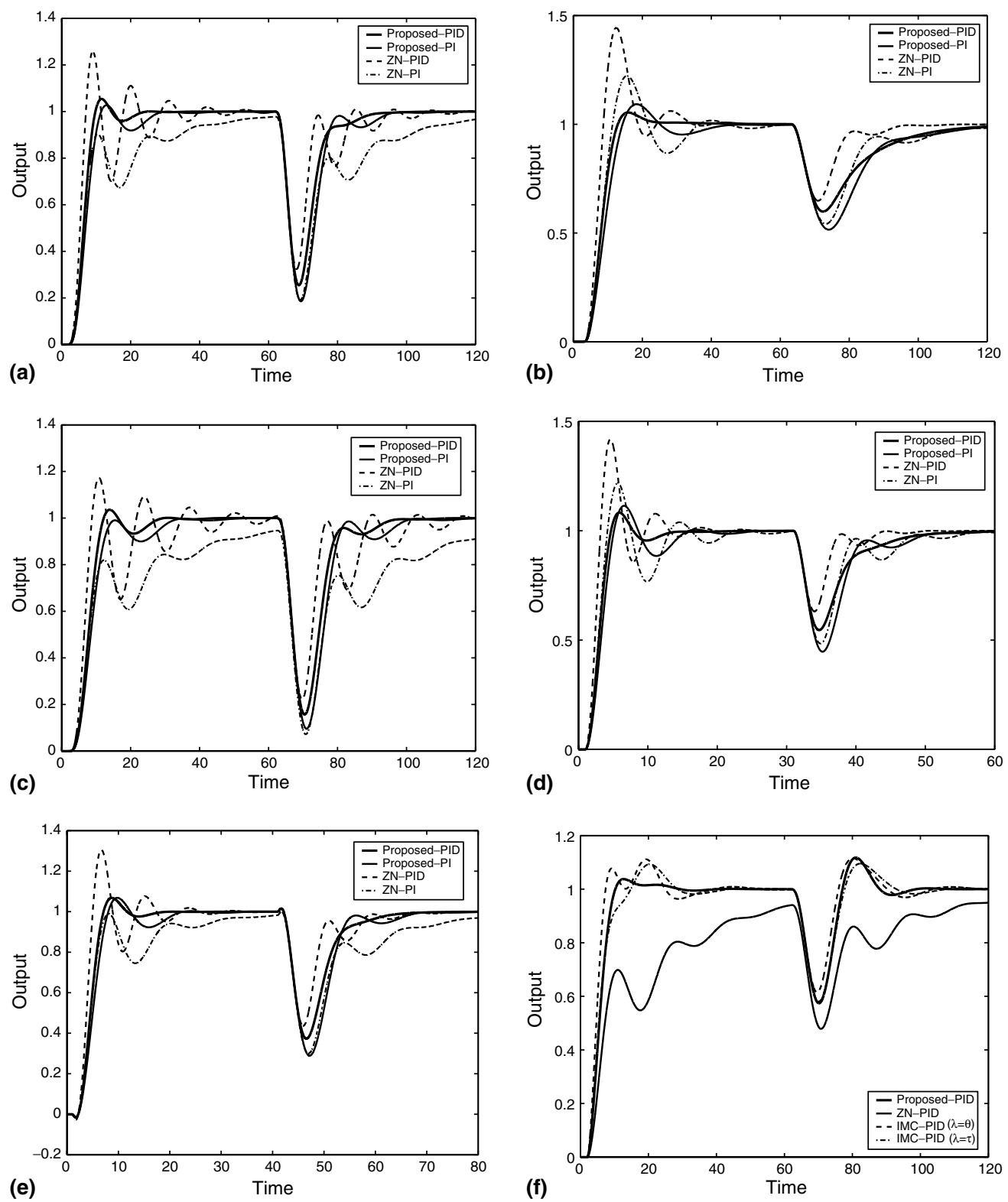

Fig. 15. Control responses of (a) Example, 1 (b) Example 2, (c) Example 3, (d) Example 4, (e) Example 5 and (f) Example 6.

the IMC tuning rules, the apparent deadtime was read directly from the initial response of the test [2]. And, after taking the deadtime, an SOPDT model for the example process is thus obtained by solving Eqs. (22) and (23) simultaneously. Based on the SOPDT model thus obtained, IMC tuning rules with different filter time constants, $\lambda$, are applied. As shown in Fig. 15(f), these two responses are obviously inferior to the one obtained from the proposed tuning procedures.

\section{Conclusions}

In this paper, a systematic approach for auto-tune of $\mathrm{PI} / \mathrm{PID}$ controller is presented. A single run of ATV experiment with a few constant cycles is carried out to identify the dynamics of the process including the model structure, steady-state gain, and an apparent dead time, in addition to conventionally obtained results such as the ultimate gain, and the ultimate frequency. Upon the results of identification, different model-based rules are provided to tune the PI/PID controllers. The advantages of this proposed method over the conventional auto-tune methods can be summarized as follows:

1. It includes the identification of model structure for choosing different tuning formula.

2. Model-based tuning rules are given in terms of $k_{\mathrm{cu}}$ and $\omega_{\mathrm{u}}$, which are popular and available from any conventional ATV experiment. 
3. Estimations of $k_{\mathrm{p}}$ and the apparent deadtime $\theta$ are obtained without carrying out additional relay feedback tests, and the estimation of $\theta$ is enhanced by using two ANNs.

4. The estimated $\theta$ is useful not only for controller tuning, but also in defining the best achievable performance. Thus, the identification proposed in this paper can be used for performance assessment as addressed by the work of Huang and Jeng [15].

The simulation results have shown that this proposed auto-tune system is efficient and self-contained.

\section{Appendix A. Derivation of the sufficient condition of $K_{\mathrm{u}}>1$ for SOPDT processes}

At ultimate frequency $\omega_{\mathrm{u}}$, we have the ultimate gain as described by Eq. (5) which can be written as:

$\tau^{4} \omega_{\mathrm{u}}^{4}+\left(4 \tau^{2} \zeta^{2}-2 \tau^{2}\right) \omega_{\mathrm{u}}^{2}+\left(1-K_{\mathrm{u}}^{2}\right)=0$

Solve the above equation for $\omega_{\mathrm{u}}^{2}$ to get

$\omega_{\mathrm{u}}^{2}=\frac{-\left(2 \zeta^{2}-1\right) \pm \sqrt{\left(2 \zeta^{2}-1\right)^{2}-\left(1-K_{\mathrm{u}}^{2}\right)}}{\tau^{2}}$

In Eq. (A.1), $\omega_{\mathrm{u}}$ must have at least one positive and real solution. Thus, when $1-2 \zeta^{2}<0$ (i.e. $\zeta>1 / \sqrt{2}$ ), the following must hold true:

$\sqrt{\left(2 \zeta^{2}-1\right)^{2}-\left(1-K_{\mathrm{u}}^{2}\right)}>2 \zeta^{2}-1$

Obviously, Eq. (A.2) is satisfied if $K_{\mathrm{u}}>1$. This means that $\zeta>(1 / \sqrt{2})$ implies $K_{\mathrm{u}}>1$. Notice that the reverse does not necessarily hold.

\section{Appendix B. Derivation of Eq. (16)}

The frequency responses at ultimate frequency $\omega_{\mathrm{u}}$ for SOPDT process are given in Eqs. (22) and (23). They can be rewritten as the following equations.

$\frac{-2 \omega_{\mathrm{u}} \tau \zeta}{1-\omega_{\mathrm{u}}^{2} \tau^{2}}=\tan \left(\omega_{\mathrm{u}} \theta\right)$

$\left(1-\omega_{\mathrm{u}}^{2} \tau^{2}\right)^{2}+\left(2 \omega_{\mathrm{u}} \tau \zeta\right)^{2}=K_{\mathrm{u}}^{2}$

From Eq. (B.1), we have $2 \omega_{u} \tau \zeta=-\left(1-\omega_{u}^{2} \tau^{2}\right) \tan \left(\omega_{u} \theta\right)$, and substitute it into Eq. (B.2) to get

$$
\begin{aligned}
& \left(1-\omega_{\mathrm{u}}^{2} \tau^{2}\right)^{2}+\left(1-\omega_{\mathrm{u}}^{2} \tau^{2}\right)^{2} \tan ^{2}\left(\omega_{\mathrm{u}} \theta\right)=K_{\mathrm{u}}^{2} \\
& \quad \Rightarrow\left(1-\omega_{\mathrm{u}}^{2} \tau^{2}\right)^{2}=\frac{K_{\mathrm{u}}^{2}}{1+\tan ^{2}\left(\omega_{\mathrm{u}} \theta\right)}=K_{\mathrm{u}}^{2} \cos ^{2}\left(\omega_{\mathrm{u}} \theta\right) \\
& \Rightarrow\left(1-\omega_{\mathrm{u}}^{2} \tau^{2}\right)= \pm K_{\mathrm{u}} \cos \left(\omega_{\mathrm{u}} \theta\right)
\end{aligned}
$$

When $\omega_{\mathrm{u}} \tau>1$, we have, from Eq. (B.1), $0<\omega_{\mathrm{u}} \theta<\pi / 2$ so that $\cos \left(\omega_{\mathrm{u}} \theta\right)>0$. Similarly, we have $\cos \left(\omega_{\mathrm{u}} \theta\right)<0$ when $\omega_{\mathrm{u}} \tau<1$. Therefore, Eq. (B.3) is only feasible for $\left(1-\omega_{\mathrm{u}}^{2} \tau^{2}\right)=-K_{\mathrm{u}} \cos \left(\omega_{\mathrm{u}} \theta\right)$ since $K_{\mathrm{u}}$ is always positive. It follows that

$\tau=\frac{\sqrt{1+K_{\mathrm{u}} \cos \left(\omega_{\mathrm{u}} \theta\right)}}{\omega_{\mathrm{u}}}$

and

$$
\begin{aligned}
2 \omega_{\mathrm{u}} \tau \zeta & =-\left(1-\omega_{\mathrm{u}}^{2} \tau^{2}\right) \tan \left(\omega_{\mathrm{u}} \theta\right) \\
& =K_{\mathrm{u}} \cos \left(\omega_{\mathrm{u}} \theta\right) \tan \left(\omega_{\mathrm{u}} \theta\right)=K_{\mathrm{u}} \sin \left(\omega_{\mathrm{u}} \theta\right) \\
& \Rightarrow \zeta=\frac{K_{\mathrm{u}} \sin \left(\omega_{\mathrm{u}} \theta\right)}{2 \omega_{\mathrm{u}} \tau}=\frac{K_{\mathrm{u}} \sin \left(\omega_{\mathrm{u}} \theta\right)}{2 \sqrt{1+K_{\mathrm{u}} \cos \left(\omega_{\mathrm{u}} \theta\right)}}
\end{aligned}
$$

The above result is the one as given in Eq. (16).

\section{References}

[1] K.J. Åström, T. Hägglund, Automatic tuning of simple regulators with specifications on phase and amplitude margins, Automatica 20 (1984) 645-651.

[2] W.L. Luyben, Derivation of transfer functions for highly nonlinear distillation columns, Ind. Eng. Chem. Res. 26 (1987) 24902495.

[3] I.L. Chien, P.S. Fruehauf, Consider IMC tuning to improve controller performance, Chem. Eng. Prog. 86 (1990) 33-41.

[4] W. Li, E. Eskinat, W.L. Luyben, An improved autotune identification method, Ind. Eng. Chem. Res. 30 (1991) 1530-1541.

[5] R.C. Chang, S.H. Shen, C.C. Yu, Derivation of transfer function from relay feedback systems, Ind. Eng. Chem. Res. 31 (1992) 855860.

[6] J. Lee, S.W. Sung, Comparison of two identification methods for PID controller tuning, AIChE J. 39 (1993) 695-697.

[7] S.H. Shen, J.S. Wu, C.C. Yu, Use of biased-relay feedback for system identification, AIChE J. 42 (1996) 1174-1180.

[8] S.H. Shen, H.D. Yu, C.C. Yu, Use of saturation-relay feedback method for autotune identification, Chem. Eng. Sci. 51 (1996) 1187-1198.

[9] Q.G. Wang, C.C. Hang, B. Zou, Low-order modeling from relay feedback, Ind. Eng. Chem. Res. 36 (1997) 375-381.

[10] C. Scali, G. Marchetti, D. Semino, Relay with additional delay for identification and autotuning of completely unknown processes, Ind. Eng. Chem. Res. 38 (1999) 1987-1997.

[11] H.P. Huang, M.W. Lee, I.L. Chien, Identification of transfer function models from the relay feedback test, Chem. Eng. Commun. 180 (2000) 231-253.

[12] I. Kaya, D.P. Atherton, Parameter estimation from relay autotuning with asymmetric limit cycle data, J. Process Contr. 11 (2001) 429-439.

[13] H.P. Huang, J.C. Jeng, Identification for monitoring and autotuning of PID controllers, J. Chem. Eng. Jpn. 36 (2003) 284-296.

[14] B.D. Tyreus, W.L. Luyben, Tuning PI controllers for integrator/ deadtime processes, Ind. Eng. Chem. Res. 31 (1992) 2625-2628.

[15] H.P. Huang, J.C. Jeng, Monitoring and assessment of control performance for single loop systems, Ind. Eng. Chem. Res. 41 (2002) 1297-1309.

[16] S.W. Sung, I.B. Lee, Enhanced relay feedback method, Ind. Eng. Chem. Res. 36 (1997) 5526-5530. 